Military Technical College

Kobry El-Kobbah, Cairo, Egypt

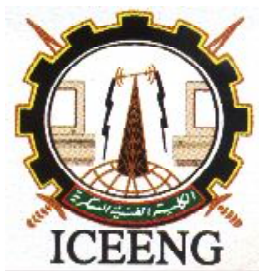

\author{
$10^{\text {th }}$ International Conference \\ on Electrical Engineering
}

ICEENG 2016

\title{
Prediction of Output Solar Power Generation using Neural Network Time Series Method
}

\author{
${ }^{1}$ Garima Sharma \\ ${ }^{1}$ M.Tech student, Dept. of Electrical Eng \\ RCEW, Jaipur \\ Rajasthan, India \\ Garimasharma.ee@gmail.com
}

\author{
${ }^{2}$ Alok Pandey and ${ }^{3}$ Pravesh Chaudhary \\ ${ }^{2,3}$ Associate Professor \\ Sine International Institute of Engineeering \\ Jaipur, Rajasthan, India \\ 2eralok_ece@yahoo.co.in, ${ }^{3}$ praveshphysics@gmail.com
}

\begin{abstract}
ANN) are used for many years to optimize the results of various problems in various sectors and disciplines like, Engineering, Industrial applications, Finance, Medical applications, Economy, Forecasts, etc. The training ability of ANN has capability to deal with nonlinear and complicated issues termed for its utilization to solve projection troubles. In this paper we have developed a nonlinear auto regressive or NARX model with exogenous input, for the forecasting of AC system output of a solar power plant placed in RCEW college jaipur (Rajasthan) for the year 2014. In this system for the purpose of forecasting, the Levenberg-Marquardt (LM) optimization approach was utilized as it aids the finest training rate pursued as a back propagation algorithm for the multilayer feed forward ANN model using MATLAB ${ }^{\circledR}$ R2013 ANN time series toolbox. The outcome of the model concluded that the preferred procedure is powerful in prediction of forthcoming future energy generation demands for the daily operational planning of solar power generation.

Keywords-Solar radiation, Forecast, Neural networks
\end{abstract}

\section{INTRODUCTION}

Solar power prediction remains an important challenge for renewable energy integration primarily due to its inherent variability and intermittency. In this work, a neural network based solar power forecasting framework is developed for the RCEW college Jaipur city.
The techniques to forecast the solar radiation can be divided in three mains groups [1]:

- Numerical models for weather prediction.

- Statistical weather forecasting models.

- Hybrid models for weather forecasting models.

The Numerical Weather Prediction models [2] are essentially based on the mathematical modeling of the atmosphere to forecast the weather based on current atmospheric conditions.

The Statistical models are based on the concept to recreate the association between the irradiance per hour and past meteorological parameters or past irradiance interpretations. Artificial Neural Networks (ANN) is the widely used models for irradiance forecast. By using this method of forecasting we can achieve quick and easy algorithms that use only local meteorological measurements [3, 4, 5] and statistical attribute parameters [6].

The Hybrid model is a combination of both Numerical models and Statistical models [7,8]. The first one is used for the forecast while the second method is used to correct the position effects through local measurements. The statistical models are essentially used to down scale the irradiance forecast. 
Forecasting is a sort of active filtering, in which future values are predicted Training Network more time. Active neural networ lines, are used for nonlinear filtering and prediction. In this paper we are predicting the output AC power of the solar power system at jaipur city in Rajasthan. Hero wo aro usine the $\mathrm{N} \Lambda \mathrm{DV}$ ANN tool for the prediction of $\mathrm{t} \quad$ Testing Network

\section{DESIGN AND METHODOLOGY}

The developed ANN model is based on one of the neural network architecture called nonlinear autoregressive with exogenous input, or NARX model. In general, now days this model is the biggest accepted network formation. In this type of time series problem, we predict the future values of a time series $z(t)$ from previous values of that time series and previous values of a second time series $r(t)$. Nonlinear autoregressive with external input, or NARX and can be written as follows:

$$
z(t)=f(z(t-1), \ldots, z(t-d), r(t-1), \ldots,(t-d))
$$

Second time series which is also known as nonlinear autoregressive, or NAR, can be expressed as here $\mathrm{z}(\mathrm{t})$ are predicted only from past values of that series

$$
z(t)=f(z(t-1), \ldots, z(t-d)
$$

The basic flow graph of designing of ANN model is shown in Fig.1.The hourly solar data, namely, Beam Irradiance $\left(\mathrm{W} / \mathrm{m}^{2}\right)$, Diffuse Irradiance (W/m2), Ambient Temperature (C) and Cell Temperature (C) of 1 year from January 2014 to December 2014 of Jaipur city collected from the National Renewable Energy Laboratory (NREL). Ambient Temperature (C) and Cell Temperature $(C)$ were used as the input data for training and testing ANN model which will be used for the prediction of hourly AC System Output.

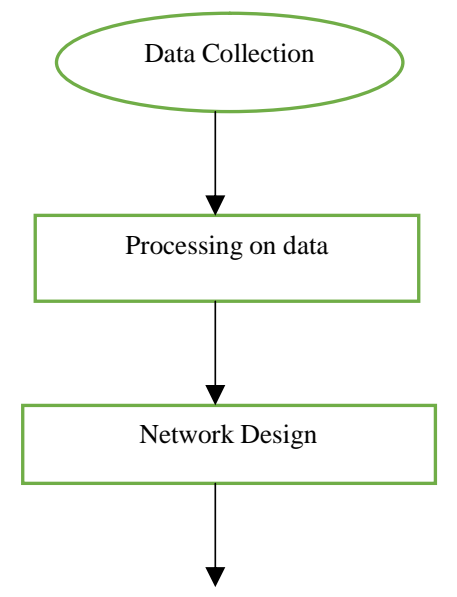

Fig.1 Flow chart of ANN model

The whole data is divided into two parts, the first set of data is collected from morning hours i.e. from 9.00 O'clock to 13:00 clock and another set of data is collected from 13.00 o'clock to 17.00O' clock. A three-layer structure, one input layer, one hidden layer, and one output layer was selected with logsigmoid transfer function for hidden layer and linear transfer function for output layer. Fig. 2 provides an overview of the structure of ANN model for the prediction of AC System Output.

The input consists of daily 24 hour ambient temperature for 12 months of the year 2014 and daily cell temperature for 365 days. The output layer will be a day's 24 hours AC solar power forecast for RCWA College, Jaipur city. The Target data is the same as the input's daily 24 hours AC solar power.

The chosen solar data were divided into two randomly selected groups, the training group, corresponding to $70 \%$ of the patterns, the validation and the test group, corresponding to $15 \%$ of patterns, so that the generalization capacity of network could be checked after training phase.

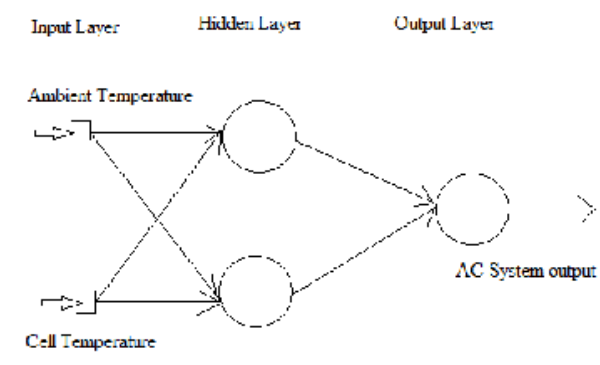

Fig.2 Architecture of ANN model

Networks were trained for a fixed number of epochs. The delays are two and the optimal number of hidden neurons that is 20 in our works was obtained experimentally by changing the network design and running the training process several times until a good performance was obtained. There is no theoretical approach to find the exact number of hidden layer neurons. This number was determined. By examining the Mean squared error (MSE) over a validation set for a varying number of hidden 
layer neurons where a number producing the smallest error was selected

The network was created and trained in close loop form as shown below in Fig. 3 .Open loop (single-step) is less efficient than closed loop (multi-step) training. Close loop allows us to supply the network with correct past outputs as we train it to produce the correct current outputs.

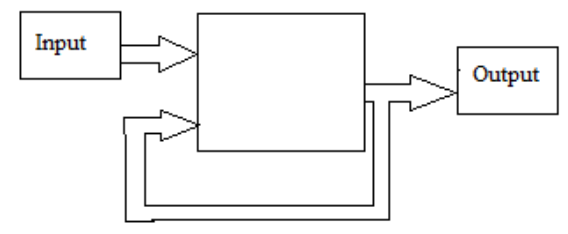

Fig.3 Architecture of NARX model

\section{THE NEURAL NETWORK TRAINING PROCESS}

The Training goal was set at 0 so as to ensure zero tolerance to network computational errors. The transfer functions used were the log-sigmoid in the Hidden layer neurons while the linear function was used in the output layer neurons. The learning function used is the Levenberg Marquardt approach. The Levenberg Marquardt training function was purposed as it holds more appropriate training progression compared to the another accessible functions in prediction issues. The maximal sum of epochs was fixed to 1000 which is the default number. Conclusively, the training scale was also fixed to the default number and left to modify accordingly as the training process increases.

\section{DISCUSSION OF TRAINING RESULTS}

This section provides a description of the conclusions obtained from the trained NARX model. This comprises the regression study chart between the output and point vectors, the typical system failure behavior and the learning phase. The three charts were formed after the entire training action is complete which include:

i. The regression plots shown in Fig.4 ii. The response of output shown in Fig.5

Iv.The Forecast and Actual Data comparison Plot shown in Fig.6

Basically the input data set was divided into three: $70 \%$ was used for as training set while $15 \%$ each was used for testing and validation of the network output results. The training data set is mandatory for attaining the neural network's weight and bias values during model training process. For systematically check the capability of the system the validation data set is needed to generalize. Lastly, the test data set is used in the evaluation of generalization error (i.e. MSE).

\section{CONCLUSIONS}

A load forecasting model was designed using Matlab R2013a ANN time series Toolbox. The implementation of the network architecture, training of the Neural Network and simulation of final conclusions were all fruitful along an extremely big level of efficiency concluding into hourly AC system output. A set of optimized weights and the associated biases after training the network from load data obtained from the power utility company were also obtained. The efficiency of the prediction was documented by equalizing the simulated outputs from the model with achieved outcomes from the utility company. Several networks architectures were trained and simulated before arriving at the best Mean squared error performance of 7.5.

\section{REFRENCES}

[1] H. M. Diagne, M. David, P. Lauret, J. Boland. Proceedings World Renewable Energy Forum WREF 2012, Denver, USA, May 2012.

[2] S.C. Muller, J. Remund. Proceedings 25th EUPVSEC Conference (2010) 4629.

[3] L. Martin, L.F. Zarzalejo, J. Polo, A. Navarro, R. Marchante, M. Cony. Solar Energy 84 (2010) 1772.

[4] A. Mellit, A. Massi Pavan. Solar Energy 84 (2010) 807.

[5] C. Voyant, P. Randimivololona, M.L. Nivet, C. Poli, M. Muselli. Meteorological Applications (2013) DOI: 10.002/met.1387.

[6] F. Wang, Z. Mi, S. Su, H. Zhao. Energies 5 (2012) 1355.

[7] R. A. Guarnieri, E.B. Pereira, S.C. Chou. Proceedings 8th ICSHMO (2006) 1777.

[8] C. Chen, S. Duan, T. Cai, B. Liu. Solar Energy 85 (2011) 2856. 

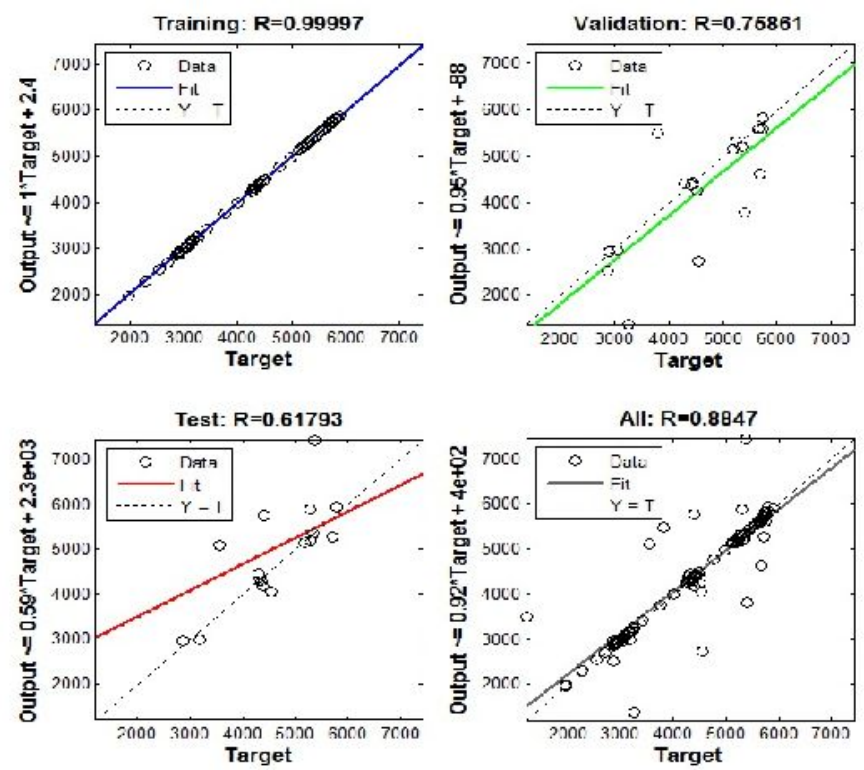

Fig.4 regression plots

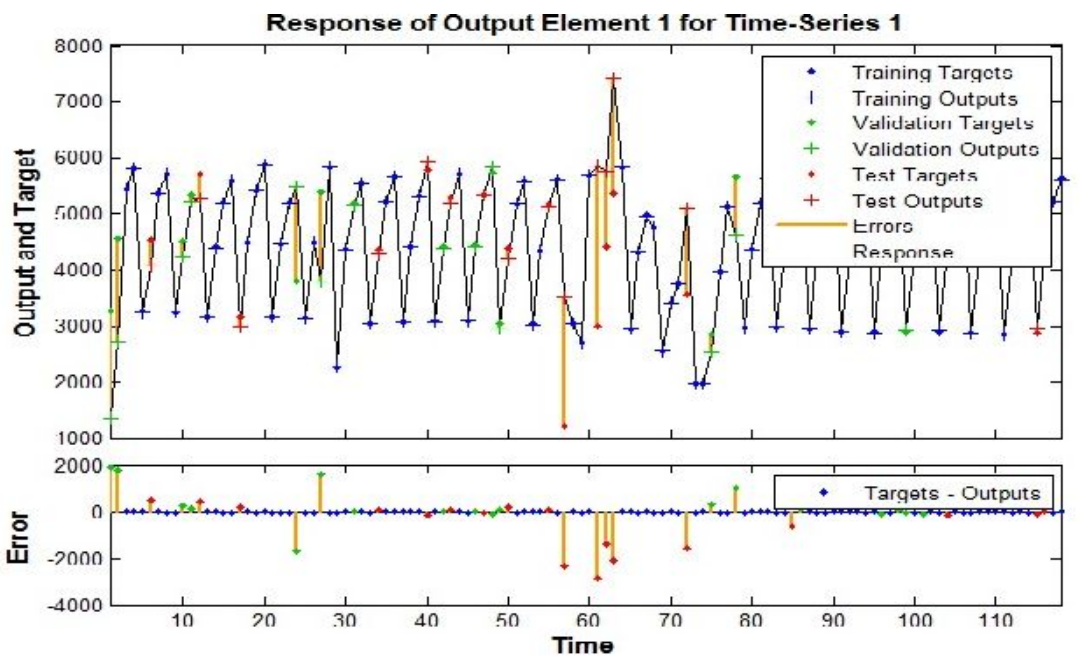

Fig.5 Response of output for time series 


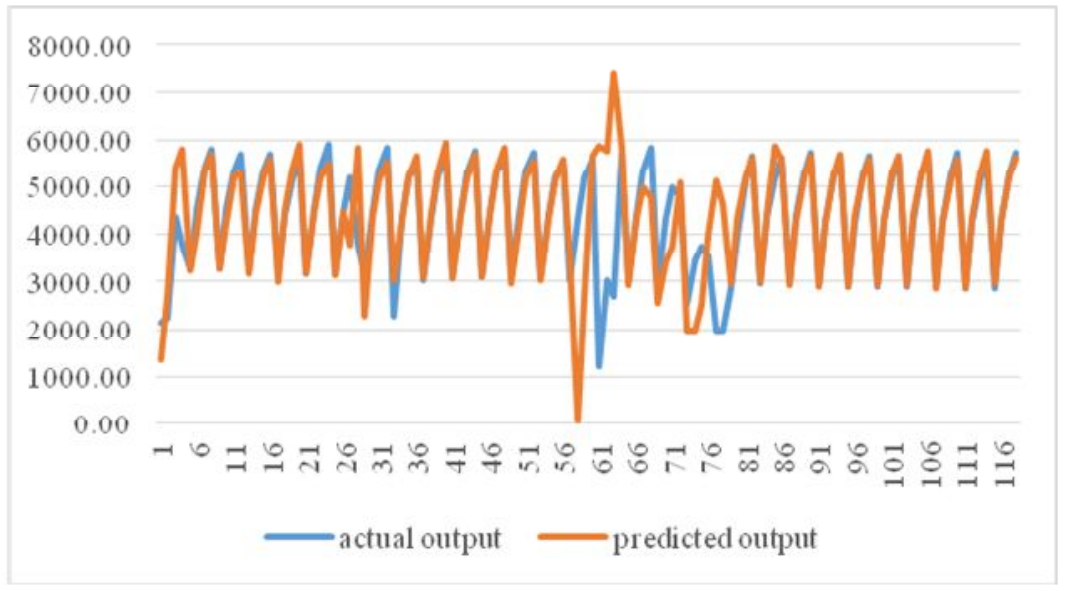

Fig. 6 Forecast and Actual Data comparison Plot 